\title{
What future in the treatment of osteochondral knee defects?
}

\author{
Riccardo D'Ambrosi ${ }^{1}$, Vincenza Ragone ${ }^{2}$, Nicola Ursino ${ }^{1}$ \\ ${ }^{1}$ IRCCS Istituto Ortopedico Galeazzi, U.O. C.A.S.C.O, Milan, Italy; ${ }^{2}$ IRCCS Policlinico San Donato, Milan, Italy \\ Correspondence to: Riccardo D’Ambrosi, MD. IRCCS Istituto Ortopedico Galeazzi, U.O. C.A.S.C.O, Milan, Italy. Email: riccardo.dambrosi@hotmail.it. \\ Provenance: This is an invited Editorial commissioned by Section Editor Jian Xu, MD, PhD (Orthopaedic Surgery and Sports Medicine, the First \\ Affiliated Hospital of Shenzhen University, Shenzhen, China). \\ Comment on: Stålman A, Sköldenberg O, Martinez-Carranza N, et al. No implant migration and good subjective outcome of a novel customized \\ femoral resurfacing metal implant for focal chondral lesions. Knee Surg Sports Traumatol Arthrosc 2018;26:2196-204.
}

Submitted Oct 25, 2018. Accepted for publication Nov 12, 2018.

doi: 10.21037/atm.2018.11.28

View this article at: http://dx.doi.org/10.21037/atm.2018.11.28

Chondral and osteochondral knee defects are common and can be found in more than $60 \%$ of patients undergoing knee arthroscopy. The choice treatment of these lesions is surgical and the number of procedures that are performed annually is constantly growing: more than 200,000 per year in the United States, with a constant increase every year. This growth is due first of all to the improvement of available biotechnologies and to the evidence that in case of lack of treatment there is a risk of progression of osteoarthritis. Articular cartilage injuries commonly occur in young, active patients with high functional demands, eager to return to a normal activity of daily life (1).

Microfracture has been considered for years the gold standard in the treatment of osteochondral knee defects. This technique was developed and published for the first time in 1994 for patients with post traumatic chondral lesions of the knee (2). Others indications for microfracture are unstable cartilage or degenerative lesions in knee without deformities. The aim of the technique, is to enhance chondral resurfacing by providing an enriched environment for tissue regeneration exploiting the regenerative potential of mesenchymal cells. Several issues remain after bone marrow stimulation procedure, in particular as regards durability of the procedure, quality of neo-cartilage, lesion size and age (2).

To overcome the disadvantages of microfracture, new treatment options have been evolved, in particular single-step scaffold-based. The ratio of scaffold is to enhance bone marrow stimulation, implanting a matrix in the damaged area to cover the blood clot. In this way articular congruence is restored by guaranteeing normal biomechanics and tribology of the joint (3).

In order for the transplant to be successful, it is crucial to create and maintain congruent articular surfaces, along with the integrated support from the underlying (3). The graft-host interference fit is a direct product of the material properties and geometries of the graft and the host implantation site. Grafts that protrude or are implanted below the joint line lead to biomechanical changes that may cause a failure in a high percentage of cases (4).

Most of the current literature reports clinical and radiological outcomes following primary cartilage surgery, but only few studies analyzed results after revision cartilage procedures due to a previously failed cartilage reconstructive procedure. Considering that failure rate in cartilage repair ranges from $14 \%$ to $43 \%$, in the next years there will be more and more evidence of revision surgery in the treatment of cartilage lesions (5).

In this scenario has gained popularity the use of focal metallic implant, both for primary or revision surgery (6). The focal metallic cartilage resurfacing is a surgical method that offers an appropriate step between regenerative procedures and replacement surgery, especially in middleaged patients with full-thickness chondral lesions. This technique allows to address the defect, to respect surrounding tissue and to provide a stability similar to replacement surgery (6).

In particular, recently, a systematic review of the literature evaluated the outcomes of focal metallic inlay resurfacing for the treatment of isolated cartilage defects of the femoral condyles concluding that this system seems to be a viable option for carefully selected patients. The review 
reported a significant improvement in knee function and pain, also if many doubts remain as regards progression of osteoarthritis (6).

Recently Stålman et al. developed a customized femoral condyle implant for focal chondral defects in order to fit precisely each patient's individual size and location of damage (7). The study analyzed results in ten patients, aged between 36 and 56 years with isolated cartilage lesions, and failed previous conservative or surgical interventions. The final follow-up was 2 years after surgery, and each patient was evaluated using using Visual Analogue Scale (VAS pain), EuroQoL (EQ-5D), Knee injuries and Osteoarthritis Outcome Score (KOOS) and radiostereometric analysis (RSA). The customized implant and guide instruments were manufactured by computer-aided design/computer-aided manufacturing (CAD/CAM) techniques using magnetic resonance imaging (MRI) data. All patients showed significant improvement as regards VAS, Tegner and the KOOS sbuscores function in daily living (ADL), sport and recreation and quality of life. No complications were reported such as infections or deep venous thrombosis. Radiologic evaluation didn't show deterioration in the tibial cartilage and none of the implants showed signs of migration (7).

The idea of customizing metal inlay is very interesting and no articles are currently present in literature. Several issues remain regarding the use of this system, especially when compared to the use of a unicompartmental knee arthroplasty (UKA). First of all the costs, in fact, the authors do not report real costs related to the production of the metal plug, but like any custom made device it needs an additive manufacturing (AM) technologies and an ad hoc design. Nowadays AM technologies allow the realization of the very complex implants using a stratum-layer strategy, starting from a 3D CAD design. AM technologies are able to produce customized implants with a greater complexity in design allowing the performance of the component to increase through its correct redesign.

Furthermore, it is not negligible to save on the processing waste in the case of use of expensive materials and to have the advantage of creating a custom-made device through a single process, saving time and costs with greater degrees of freedom in design of implants.

In this regard a recent article analyzed the value for money of UKA in England and Wales, highlighting that in selected patients, UKA provides an alternative to total knee replacement (TKR) that is less costly for the healthcare system providing better health outcomes over their lifetime.
If surgeons performing UKA achieved sufficient usage of the procedure, future economic and population health gains would likely be increased even further (8).

Furthermore, literature in recent years has shown that the use of patient specific instruments (PSI) for the patient cannot be considered an advantage, if not in less experienced surgeons. In fact, a 2018 article underlined how in a considerable number of cases there was no agreement between the manufacturer's and the surgeon's pre-operative plans, especially with regard to tibial components (9). A similar incident also occurred in the work of Stålman et al. in which in one patient $(10 \%)$ MRI underestimated the size of the injury and the implant was too small to fit the damaged site (7).

Even a work by Leenders et al. noted that following the UKA with specific instrumentation the most reported complication was the tibia fracture $(3.3 \%)(10)$. The use of specific instruments should be reserved in particular for young surgeons with a not yet complete learning curve, particularly in UKA; in fact, Jones et al. have shown how in a sawbone model, PSI allowed inexperienced surgeons to achieve more accurate saw cuts, equivalent to expert surgeons, and thus has the potential to reduce revision rates (11).

Stalman et al. decide to treat his patients between the ages of 30 and 65 using the metal implant instead of an arthroplasty, claiming that knee replacement surgery have a high risk of early failure in young patients (7). In recent years, literature has changed its mind about this, in fact recently Greco et al. reviewed 340 patients who underwent UKA with an average age of 46.5 years (12). Patients reported significant improvements in knee function, University of California Los Angeles activity score, Knee Society clinical and functional scores with a survival rate of $96 \%$ at 6 years and $86 \%$ at 10 years. Similar results were reported by Faour Martín et al. in which survival rates in patients younger than 60 years were $95 \%$ (13).

Moreover, Stålman et al. excluded from the study all patients with a BMI $>35 \mathrm{~kg} / \mathrm{m}^{2}$ (7); considering that obesity is projected to increase all around the world, this could be a huge limitation (14). As reported by Plate, this is not a contraindication in UKA implant, in fact the author noted that patient's BMI has no correlation with the rate of revision surgery (15). Furthermore, BMI did not influence 90-day readmissions but showed significant correlation with higher opioid medication requirements and a higher number of physical therapy (15). These results are confirmed also by Lum et al. stating that severely obese patients who underwent medial UKA demonstrated similar 
Table 1 Algorithm treatment for osteochondral knee defects after previous failed conservative treatment and surgical treatment

\begin{tabular}{|c|c|c|}
\hline Age, years & \multicolumn{2}{|c|}{ BMI, $\mathrm{kg} / \mathrm{m}^{2}$} \\
\hline \multicolumn{3}{|c|}{ Failed conservative treatment } \\
\hline \multirow[t]{2}{*}{$>55$} & UKA (+) & UKA (+/-) \\
\hline & Resurfacing metal implant (-) & Resurfacing metal implant (+) \\
\hline$<55$ & Resurfacing metal implant (+/-) & Resurfacing metal implant (+) \\
\hline \multicolumn{3}{|c|}{ Failed surgical treatment } \\
\hline \multirow[t]{2}{*}{$>55$} & \multicolumn{2}{|c|}{ UKA (+) } \\
\hline & \multicolumn{2}{|c|}{ Resurfacing metal implant (-) } \\
\hline
\end{tabular}

-, not recommended treatment; +/-, questionable treatment; +, choice treatment. UKA, unicompartmental knee arthroplasty.

survivorship with substantially fewer reoperations, reduced deep infection, and less perioperative complications (16).

Moreover, important aspect to evaluate after osteochondral knee injury is the ability to return to sports, especially in young patients with high functional demands. Stålman et al. reported a significant improvement at 2-year follow-up as regards Tegner and sport and recreation (7). A review performed by Waldstein et al. analysed sport and physical activity following UKA reporting a return to sports activity ranging from $87 \%$ to $98 \%$ (17). All patients were evaluated using the UCLA activity score, the Tegner activity score or the High Activity Arthroplasty Score, respectively. The return to activity rate ranged from (17).

Another aspect to analyze is the failure of customize inlay implant: in these situations, it would be interesting to evaluate if the replacement surgery is more difficult than a first implant, analyzing the surgical times and the patient's blood loss.

A last consideration regards surgical technique and instrumentations: in two patients the author reported that the lesion was too lateral and close to patella, and to solve the problem the drillguide was cut (7). UKA in recent years have had considerable improvements with regard to instrumentation, significantly reducing surgical times and allowing a more efficient procedure $(18,19)$.

In light of the fact that there is still no gold standard in the literature regarding the use of metal inlay or UKA we proposed a treatment algorithm that simplifies the choice of therapies for femoral condyle injuries (Table 1).

\section{Acknowledgements}

None.

\section{Footnote}

Conflicts of Interest: The authors have no conflicts of interest to declare.

\section{References}

1. Rodriguez-Merchan EC. Regeneration of articular cartilage of the knee. Rheumatol Int 2013;33:837-45.

2. Erggelet C, Vavken P. Microfracture for the treatment of cartilage defects in the knee joint - A golden standard? J Clin Orthop Trauma 2016;7:145-52.

3. Filardo G, Kon E, Roffi A, et al. Scaffold-based repair for cartilage healing: a systematic review and technical note. Arthroscopy 2013;29:174-86.

4. Bowland P, Ingham E, Jennings L, et al. Review of the biomechanics and biotribology of osteochondral grafts used for surgical interventions in the knee. Proc Inst Mech Eng H 2015;229:879-88.

5. Lamplot JD, Schafer KA, Matava MJ. Treatment of Failed Articular Cartilage Reconstructive Procedures of the Knee: A Systematic Review. Orthop J Sports Med 2018;6:2325967118761871.

6. Malahias MA, Chytas D, Thorey F. The clinical outcome of the different HemiCAP and UniCAP knee implants: A 
systematic and comprehensive review. Orthop Rev (Pavia) 2018;10:7531.

7. Stålman A, Sköldenberg O, Martinez-Carranza N, et al. No implant migration and good subjective outcome of a novel customized femoral resurfacing metal implant for focal chondral lesions. Knee Surg Sports Traumatol Arthrosc 2018;26:2196-204.

8. Burn E, Liddle AD, Hamilton TW, et al. Costeffectiveness of unicompartmental compared with total knee replacement: a population-based study using data from the National Joint Registry for England and Wales. BMJ Open 2018;8:e020977.

9. Cucchi D, Menon A, Compagnoni R, et al. Significant differences between manufacturer and surgeon in the accuracy of final componentsize prediction with CT-based patient-specific instrumentation for total knee arthroplasty. Knee Surg Sports Traumatol Arthrosc 2018;26:3317-24.

10. Leenders AM, Schotanus MGM, Wind RJP, et al. A high rate of tibial plateau fractures after early experience with patient-specific instrumentationfor unicompartmental knee arthroplasties. Knee Surg Sports Traumatol Arthrosc 2018;26:3491-8.

11. Jones GG, Logishetty K, Clarke S, et al. Do patientspecific instruments (PSI) for UKA allow non-expert surgeons to achieve the samesaw cut accuracy as expert surgeons? Arch Orthop Trauma Surg 2018;138:1601-8.

12. Greco NJ, Lombardi AV Jr, Price AJ, et al. Medial Mobile-Bearing Unicompartmental Knee Arthroplasty in

Cite this article as: D'Ambrosi R, Ragone V, Ursino N. What future in the treatment of osteochondral knee defects? Ann Transl Med 2018;6(Suppl 2):S100. doi: 10.21037/atm.2018.11.28
Young Patients Aged Less Than or Equal to 50 Years. J Arthroplasty 2018;33:2435-9.

13. Faour Martín O, Valverde García JA, Martín Ferrero MÁ, et al. The young patient and the medial unicompartmental knee replacement. Acta Orthop Belg 2015;81:283-8.

14. Pineda E, Sanchez-Romero LM, Brown M, et al. Forecasting Future Trends in Obesity across Europe: The Value of Improving Surveillance. Obes Facts 2018;11:360-1.

15. Plate JF, Augart MA, Seyler TM, et al. Obesity has no effect on outcomes following unicompartmental knee arthroplasty. Knee Surg Sports Traumatol Arthrosc 2017;25:645-1.

16. Lum ZC, Crawford DA, Lombardi AV Jr, et al. Early comparative outcomes of unicompartmental and total knee arthroplasty in severely obese patients. Knee 2018;25:161-6.

17. Waldstein W, Kolbitsch P, Koller U, et al. Sport and physical activity following unicompartmental knee arthroplasty: a systematic review. Knee Surg Sports Traumatol Arthrosc 2017;25:717-28.

18. Jang KM, Lim HC, Han SB, et al. Does new instrumentation improve radiologic alignment of the Oxford® medial unicompartmental knee arthroplasty? Knee 2017;24:641-50.

19. Tu Y, Xue H, Ma T, et al. Superior femoral component alignment can be achieved with Oxford microplasty instrumentation after minimally invasive unicompartmental knee arthroplasty. Knee Surg Sports Traumatol Arthrosc 2017;25:729-35. 